\title{
Analytic Expressions for Displacement Fields of Dislocation Loops in Anisotropic Cubic Crystals
}

\author{
By H. Scheuer, P. H. Dederichs and W. Schmatz \\ Institut für Festkörperforschung der Kernforchungsanlage Jülich, Postfach 365, 517 Jülich, Germany (BRD)
}

(Received 29 April 1974)

\begin{abstract}
Based on the analytic expression for the elastic Green's function of anisotropic cubic crystals and on the description of a dislocation by a special distribution of dipole forces the displacement field of a dislocation loop is represented by a line integral. With the displacement field one can give analytic expressions for the dilation and the stress tensor in reciprocal space. From this, cross sections for Huang scattering and for nuclear and magnetic small-angle scattering at dislocations can be calculated. In the case of nuclear and magnetic scattering at straight dislocations, values were found which differ by a factor 0.5 to 5 from the values expected with the assumption of elastic isotropy.
\end{abstract}

J. Appl. Cryst. (1975). 8, 143

\section{Diffuse X-ray Scattering from the Displacement Field of Point Defects and Defect Clusters}

\author{
BY H.PEISL* \\ Physik Department der Technischen Universität München, 8046 Garching, Germany (BRD)
}

(Received 14 August 1974; accepted 24 October 1974)

\begin{abstract}
This review gives first a short introduction to the theoretical relations between experimentally observed diffuse scattering results and the desired information about defects. The situation is discussed under which diffuse scattered X-ray intensity from defects can be distinguished from other diffuse intensities (thermal diffuse scattering, Compton scattering). A typical experimental set-up is described to show what the requirements are in experimental resolution and how intensity distribution in reciprocal space can be measured most conveniently. A few typical experiments are discussed to demonstrate the physical potential of this method for studying impurities in metals and radiation-induced defects and defect clusters in ionic crystals. Special emphasis is given to the comparison of the experimental results with theoretical predictions; the intensity close to the Bragg peaks (Huang scattering) falls off as $1 / g^{2}, g$ being the distance from a reciprocal-lattice point $G$. The scattering intensity goes as $G^{2}$. The intensity distribution in reciprocal space gives typical isointensity curves from which the symmetry of the double force tensor and its components can be deduced. Together with the measured shift of a Bragg peak, measurement of absolute scattering intensity gives a unique way of determining the defect concentration. Further away from the Bragg peaks (asymptotic scattering) the intensity is proportional to $1 / g^{4}$ and shows characteristic oscillations.
\end{abstract}

\section{Introduction}

Diffuse X-ray scattering measurements are a powerful tool for studying lattice distortions by defects. Although the technique was theoretically predicted long ago, only recently have experiments shown its real power. A review of the literature has been given by Krivoglaz (1969), Schmatz (1970), Dederichs (1973) and Peisl \& Trinkaus (1973).

This review gives first a short introduction to the

* Present adress: Sektion Physik der Ludwig-Maximilians Universität München, 8000 München, Germany (BRD). theoretical relations between experimental results and the desired information $(\S 2)$. In $\S 3$ we discuss the situations under which diffuse scattered X-ray intensity from defects can be distinguished from other diffuse intensities. $\S 4$ describes a typical experimental set-up in order to show the requirements in experimental resolution and to show how intensity distributions in reciprocal space can be obtained most conveniently. In $\S 5$ a few typical experiments will be discussed to demonstrate the physical potential of this method for studying impurities in metals, and radiation-induced defects and defect clusters in metals and ionic crystals. Special emphasis will be given to the comparison of the experimental results with theoretical predictions. 